\title{
Influence of Spectral Diffusion on the Line Shapes of Single CdSe Nanocrystallite Quantum Dots
}

\author{
S. A. Empedocles and M. G. Bawendi* \\ Department of Chemistry and Center for Materials Science and Engineering, \\ Massachusetts Institute of Technology, Cambridge, Massachusetts 02139
}

Received: August 6, 1998; In Final Form: December 31, 1998

\begin{abstract}
We study the emission line shapes of single CdSe nanocrystallite quantum dots. Single dot line shapes are found to result from rapid spectral shifting of the emission spectrum rather than the intrinsic physics of the quantum dot. A strong dependence of single dot line widths on excitation intensity, wavelength, temperature, and integration time is found and is correlated with the number of times that the quantum dot is excited during the acquisition of a single spectrum. The observed results are consistent with thermally assisted spectral diffusion, activated by the release of excess excitation energy.
\end{abstract}

Recent advances in the detection of single molecules have been responsible for a level of understanding in condensed phase systems that was not previously possible. Of particular interest are incoherent dynamic effects, which can be completely hidden when averaged over an ensemble. In recent years, single chromophore spectroscopy has allowed the direct observation of single enzyme reactions, ${ }^{1}$ single energy transfer events, ${ }^{2}$ and single molecule rotational dynamics. ${ }^{3}$ In addition, new and unexpected physical phenomena have been observed, which appear to be common to many single chromophore systems, such as fluorescence blinking ${ }^{4-6}$ and spectral shifting (spectral diffusion). ${ }^{7-12}$

One field that has greatly benefited from single chromophore spectroscopy is the study of semiconductor quantum dots (QDs). QDs are of great interest due to their unique size dependent optical properties, ${ }^{13}$ which can easily be tuned during fabrication. ${ }^{14}$ Unfortunately, the characteristics that make QDs interesting also make them inherently difficult to study. Inhomogeneities in size and shape within ensemble samples result in spectral broadening that is many orders of magnitude larger than single QD spectra. ${ }^{9,15}$ Though the mechanisms are thought to be quite different, the study of single QDs has revealed phenomena common to other single chromophore systems such as blinking ${ }^{4}$ and spectral diffusion..$^{9-12}$ It has also revealed new characteristics specific to QDs such as ultranarrow transition line widths, ${ }^{9,15}$ giant Overhauser shifts, ${ }^{16}$ multicarrier effects, ${ }^{17,18}$ and fluctuating local electric fields. ${ }^{12}$

One area of particular interest which can, in principle, be addressed on the single QD level, is the nature of the homogeneous line shape. While theory predicts that QDs should have atomic-like spectral transitions due to long excited-state lifetimes and weak coupling to acoustic phonons, ${ }^{19}$ previous ensemble experiments have suggested that line widths in both excitation $^{20-22}$ and emission ${ }^{23-25}$ are quite broad (for example, "homogeneous" line widths extracted from fluorescence line narrowing experiments in CdSe nanocrystallite QDs are reported to be $\sim 5 \mathrm{meV}^{26}$ ). It was expected that single QD spectroscopy would uncover the true "homogeneous" line width. However, while single dot line widths are typically found to be significantly narrower than what is seen in ensemble experiments, many single dot experiments still reveal line widths that are much broader than predicted, with non-Lorenzian line shapes that are strongly dependent on experimental conditions. ${ }^{9-12,27}$ For example, a range of single dot line widths over 2 orders of magnitude have been reported for II-VI nanocrystallite QDs. ${ }^{9-11}$ It has therefore been difficult to use single dot line shapes to learn about the intrinsic physics of these QD systems.

The observed variation in single dot line shapes is similar to what is seen in single molecule spectroscopy where line widths are thought to result from spectral diffusion on a time scale that is fast compared to the acquisition time of the experiment. ${ }^{28,29}$ While the mechanism of spectral diffusion is thought to be quite different in single QDs than in single molecules, a similar theory has been proposed to account for the observed line shape differences in the case of single nanocrystallite QDs. ${ }^{9}$ However, while spectral diffusion has been observed in single nanocrystallites, only indirect evidence exists regarding its contribution to single dot line shapes.

In this Article, the contribution of spectral diffusion to the line shape of single CdSe nanocrystallite QDs is demonstrated through the direct observation of spectral shifting on a $0.1 \mathrm{~s}$ time scale. In keeping with previous single nanocrystallite results, we describe these small spectral shifts in terms of Stark shifts that result from fluctuating local electric fields produced by the movement of charge carriers around individual QDs. Changes in line width as a function of excitation intensity, wavelength, integration time, and sample temperature are also analyzed and are found to be consistent with the activated movement of external charges in response to thermal and excess excitation energy released following each photoexcitation.

Single domain wurtzite nanocrystallites with an average diameter of $56.5 \AA$ were synthesized as in ref 14 . Half of the sample was then overcoated with a $\sim 7 \AA$ layer of $\mathrm{ZnS} .{ }^{30}$ The addition of a $\mathrm{ZnS}$ shell has been shown to have a significant effect on the luminescence from ensemble CdSe nanocrystallite samples, resulting in an increase in the quantum yield to as high as $50 \%$ at room temperature.

Single QD spectra were taken using a far-field epifluorescence microscope ${ }^{9}$ with $514 \mathrm{~nm}$ excitation. An extremely dilute solution of QDs (in $0.1 \%$ PMMA/toluene by weight) was spin coated onto a crystalline quartz substrate, which was then placed in a liquid helium cryostat. The sample concentration was chosen to produce an areal density of $\sim 1 \mathrm{QD} / \mu \mathrm{m}^{2}$ in order to allow individual QDs to be spatially resolved using standard far-field 

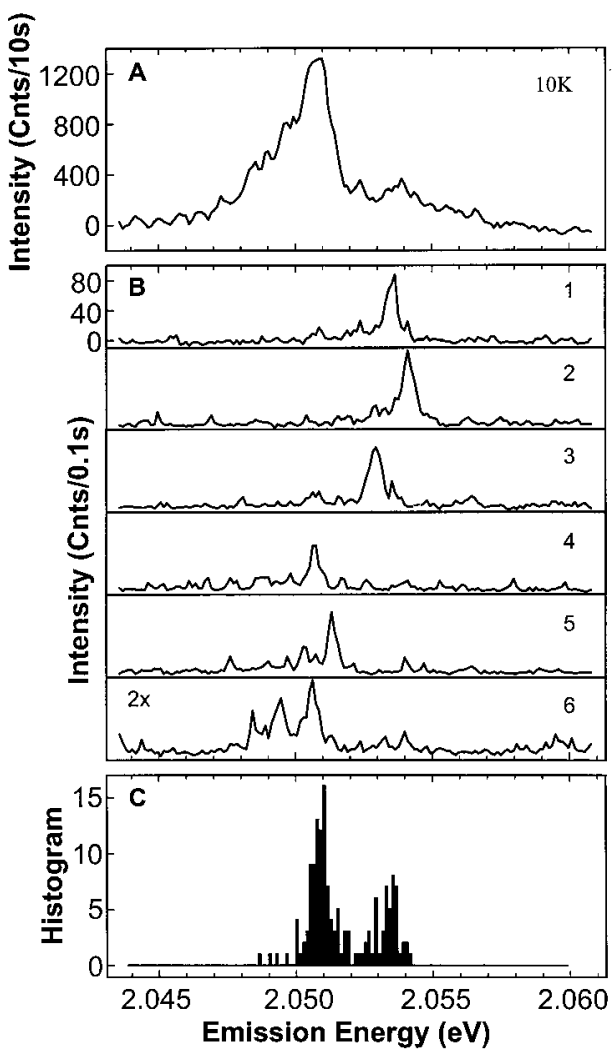

Figure 1. (A) Spectrum of a single overcoated dot with a $10 \mathrm{~s}$ integration time at $10 \mathrm{~K}$. (B) A representative sample of 6 spectra from the same dot as in (A), with a $0.1 \mathrm{~s}$ integration time. Of the 150 spectra taken at $\sim 10$ frames/s, frames 1-6 indicate spectrum number 1, 16, $35,59,84$, and 150 , respectively. Each peak in frames $1-5$ corresponds to $\sim 20$ collected photons. (C) Histogram of peak positions from each of the 150 spectra described in (B). The excitation intensity for all spectra in Figure 1 was $200 \mathrm{~W} / \mathrm{cm}^{2}$. Data in Figure 1 (only) were obtained using an intensified frame transfer CCD camera (Princeton Instruments Pentamax ICCD) with a sensitivity of $\sim 20$ counts per detected photon.

optics. Temperature data were taken on different days with fresh samples to ensure no degradation with time. Intensity and time series were taken in nonsequential order to ensure that the observed spectral changes were not caused by light-induced degradation. For each QD included in a time or intensity series, data were taken at all points within the series. Line widths were determined by direct measurement of the full width at halfmaximum (fwhm) of each peak.

A typical single dot spectrum from the overcoated sample, taken with a $10 \mathrm{~s}$ integration time, can be seen in Figure 1A (previous single nanocrystallite studies have used integration times ranging from $30 \mathrm{~s}$ to $10 \mathrm{~min}^{9-12}$ ). The observed peak, which corresponds to the zero phonon transition, has a FWHM of $\sim 2 \mathrm{meV}$ and appears to have some additional structure that is blue shifted by $\sim 3 \mathrm{meV}$ from the main peak. Spectra of the same QD taken with 100 times shorter integration time $(0.1 \mathrm{~s})$ reveal a single peak with no sideband that is much narrower than the spectrum in Figure 1A. In 150 of these fast spectra taken in rapid succession, this resolution-limited peak can be seen shifting in energy over the entire $10 \mathrm{~s}$ spectrum (Figure 1B). ${ }^{31}$ Shifting below our resolution limit may also be occurring and multipeak spectra such as frame 6 of Figure 1B indicate spectral shifting on a sub $0.1 \mathrm{~s}$ time scale.

Figure 1C shows a histogram of peak positions for the 150 spectra described above. As can be seen, the $10 \mathrm{~s}$ intensity distribution is well reproduced. In this way, we can directly
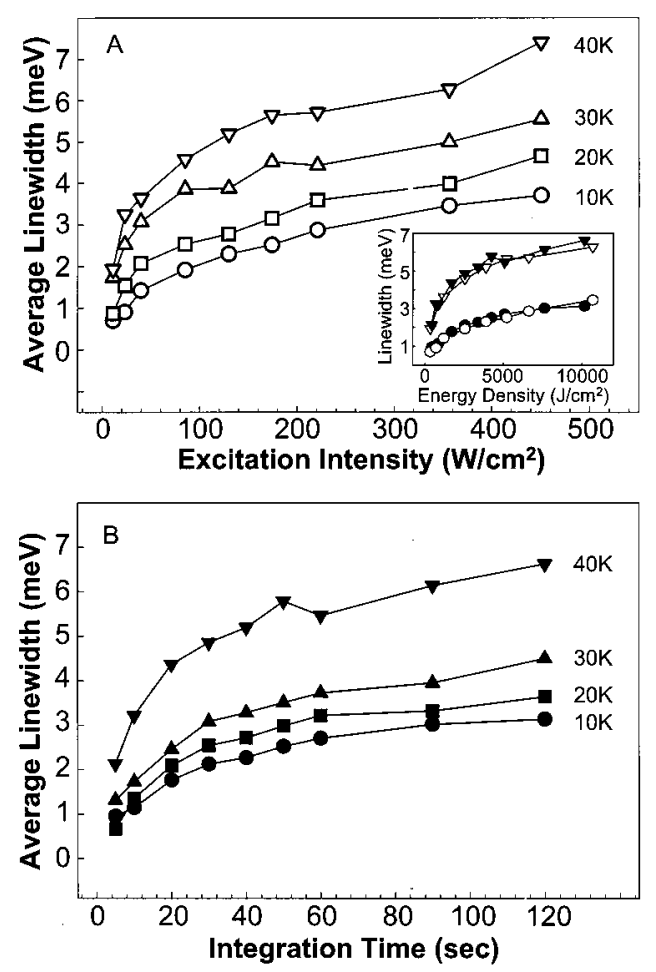

Figure 2. (A) Average single dot line width as a function of excitation intensity and temperature for the overcoated sample. Circles $(10 \mathrm{~K})$, squares $(20 \mathrm{~K})$, upward triangles $(30 \mathrm{~K})$, and downward triangles $(40 \mathrm{~K})$ represent the average line widths of 34,53 , 38, and 24 dots, respectively. Data were taken with an integration time of $30 \mathrm{~s}$. (B) Average single dot line width for the same sample as a function of integration time and temperature. Circles (10 K), squares (20 K), upward triangles $(30 \mathrm{~K})$, and downward triangles $(40 \mathrm{~K})$ represent the average line widths of $40,47,38$, and 23 dots, respectively. Data were taken with an excitation intensity of $85 \mathrm{~W} / \mathrm{cm}^{2}$. (Inset) Time and intensity data from (A) and (B) (open and closed symbols, respectively) at 10 and $40 \mathrm{~K}$ (circles and downward triangles respectively). Inset data are plotted as a function of excitation energy density (time $x$ intensity) in order to normalize for the total number of absorbed photons per spectrum.

relate the effects of spectral diffusion to the observed line shape of a single QD. Even the apparent sideband in Figure 1A is found to be an artifact of spectral diffusion. A wide variety of different single dot spectra including multiplet line shapes as well as peaks as broad as $10 \mathrm{meV}$ have been analyzed in this same manner. These data suggest that on the time scale of several seconds, the primary contribution to single dot line shapes is spectral diffusion.

Figure 2 plots the average line width of single overcoated QDs as a function of excitation intensity, integration time, and sample temperature. Figure 3 plots the average line width as a function of excitation intensity for both overcoated and nonovercoated samples.

Figures 2 and 3 demonstrate the strong dependence of single dot line widths on experimental and sample conditions, respectively. This may explain the wide range of line widths that have been reported in single nanocrystallite experiments. ${ }^{9-11}$ Reported differences are consistent with variations in experimental procedures as well as sample preparation.

It should be noted that the average line widths at the lowest intensities and shortest integration times in Figures 2 and 3 are inflated due to many individual spectra falling below the resolution limit of our spectrometer $(\sim 0.4 \mathrm{meV})$. In addition, while the average line width curves show a smooth saturation character, many individual QDs have a much more linear change 


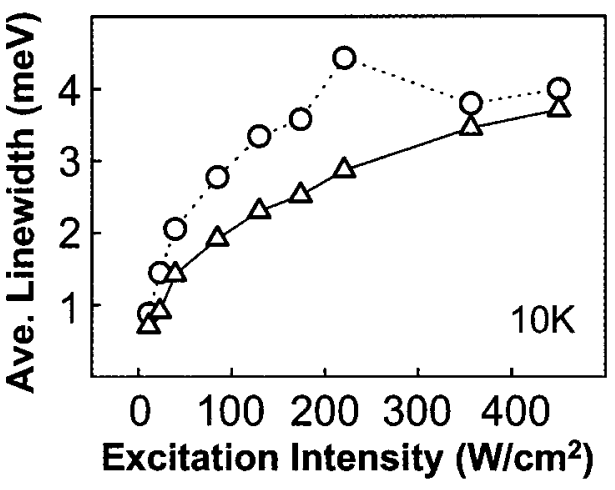

Figure 3. Average single dot line width for 34 overcoated dots and 25 nonovercoated dots (triangles and circles, respectively) as a function of excitation intensity at $10 \mathrm{~K}$. The integration time was $30 \mathrm{~s}$.

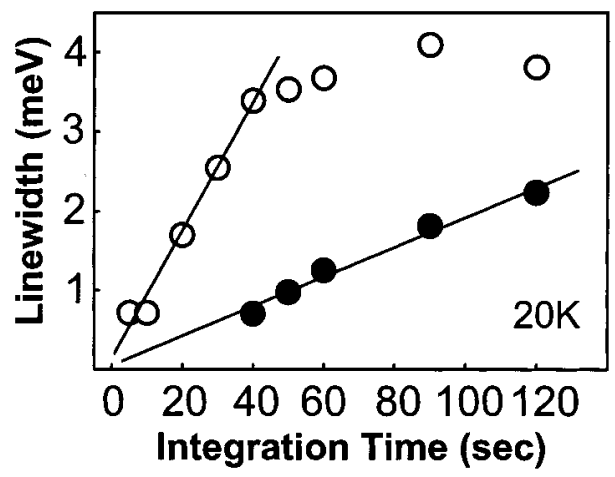

Figure 4. Line width as a function of integration time for two different single dots from the overcoated sample at $20 \mathrm{~K}$. Lines indicate a leastsquares fit to the linear region approaching the origin.

in width as a function of time and intensity, followed by a region of saturation (Figure 4). The smooth average curves are the result of differences in the slope and saturation line width of individual QDs that represent the effects of different local environments on spectral diffusion.

Electric field studies of single CdSe nanocrystallites have suggested that spectral diffusion is the result of changing local electric fields ${ }^{12}$ resulting from charges on the surface of the QD. Small fluctuations in this local field may contribute to observed single dot line widths by producing Stark shifts in single QD spectra. The line width difference between overcoated and nonovercoated QDs seen in Figure 3 is consistent with this hypothesis. The electric field studies described above found that for QDs with the same size CdSe core, a smaller average internal electric field resulting from trapped charges was found in overcoated dots than in nonovercoated samples. This difference is likely to be the result of additional screening of the local electric field by the $\mathrm{ZnS}$ shell. Also, the presence of the $\mathrm{ZnS}$ shell forces trapped charges to reside farther away from the center of the QD, further reducing the internal electric field. For electric fields on the order of the local fields measured in these experiments, the dependence of the Stark shift $(\Delta E)$ on electric field $(F)$ was found to be in the quadratic regime $(\Delta E$ $\propto F^{2}$ ). This implies that the magnitude of the change in energy $[\Delta(\Delta E)]$, and therefore the observed line width, due to a fluctuating field $(\Delta F)$ will also depend on the average field present $[\Delta(\Delta E) \propto F(\Delta F)]$. Therefore, in the presence of similar environmental fluctuations, overcoated QDs, with a smaller average internal field, should have narrower diffusion-induced line widths than nonovercoated dots. This is consistent with Figure 3.

Spectral diffusion in single molecules is typically attributed to changes in the host matrix (represented by a system of double welled potentials), which interact with individual chromophores through short-range strain fields. ${ }^{29}$ The fluctuating electric fields responsible for spectral diffusion in CdSe nanocrystallites are thought to result from the presence of charge carriers trapped on or near the surface of individual QDs $^{12}$ that result from ionization of the CdSe core. ${ }^{6,32}$ In what follows, we speculate that spectral diffusion in single nanocrystallite QDs can be described in terms of point charges in a system of many welled potentials (trap sites). Small fluctuations in the local electric field result from individual charges moving between different trap sites. As a result, single dot line shapes are determined by the number and type of external charges, the number and depth of available trap sites, and the frequency and pattern of shifts between sites during the acquisition of a single spectrum.

The time dependence of the average line width observed in Figure 2 is consistent with spectral diffusion. The intensity dependence, which is not the result of traditional power broadening since excitation occurs far from the emitting state ( $\sim 350 \mathrm{meV}$ ), can also be understood within the framework of spectral diffusion. The data in Figure 2 suggests that the observed broadening is actually related to the number of excitations that occur during the acquisition of a given spectrum. The inset of Figure 2 shows the time data (at fixed intensity) and intensity data (at fixed time) for two temperatures plotted as a function of the time-integrated intensity (energy density $=$ time $\times$ intensity). Plotted in this way, it is easy to see that changing the excitation intensity has the same effect as changing the integration time by the same relative amount. Assuming that we are in the linear absorption regime $\left(<100 \mathrm{~kW} / \mathrm{cm}^{2}\right),{ }^{33}$ this implies that it is the number of absorbed photons per spectrum that is related to the observed broadening. It therefore follows that excess excitation energy, released as the exciton relaxes to the emitting state, may be responsible for the movement of external charges. As a test of this hypothesis, the line widths of a sample of overcoated dots were studied with $514 \mathrm{~nm}$ excitation and with $573 \mathrm{~nm}$ excitation from an $\mathrm{Ar}^{+}$pumped dye laser. In this experiment, time, temperature, and the number of excitations $^{34}$ were held constant. Data for all QDs were collected at both wavelengths and the change in line width for each dot was measured. For the 126 dots studied, decreasing the excitation energy caused the average line width to decrease by $25 \%$. This suggests that the extent of spectral diffusion depends on the amount of energy released upon each excitation. This, combined with the overlap of the time and intensity curves, implies that the line shape of a single dot spectrum depends on the number of excitations and therefore the amount of energy released during its acquisition. A second implication of these data is that the energy responsible for the movement of external charges results from the excess excitation energy released as the exciton relaxes to the emitting state and not energy released via nonradiative relaxation. If nonradiative relaxation energy was an important contribution to the observed line widths, the small change in excess energy resulting from the change in excitation wavelength $(\sim 250 \mathrm{meV})$ would have a negligible effect on the observed line widths relative to the large $(\sim 2400$ $\mathrm{meV}$ ) nonradiative contribution. The fact that this small change in energy results in a significant change in line width indicates that the energy involved in spectral diffusion is small, consistent with the energy released as the exciton relaxes to the emitting state.

The effect of excess excitation energy is not the result of an isotropic increase in the temperature of the QD. Heating is expected to be insignificant in these experiments due to rapid dissipation of heat into the surrounding matrix $\left(\sim 10^{5}\right.$ excita- 

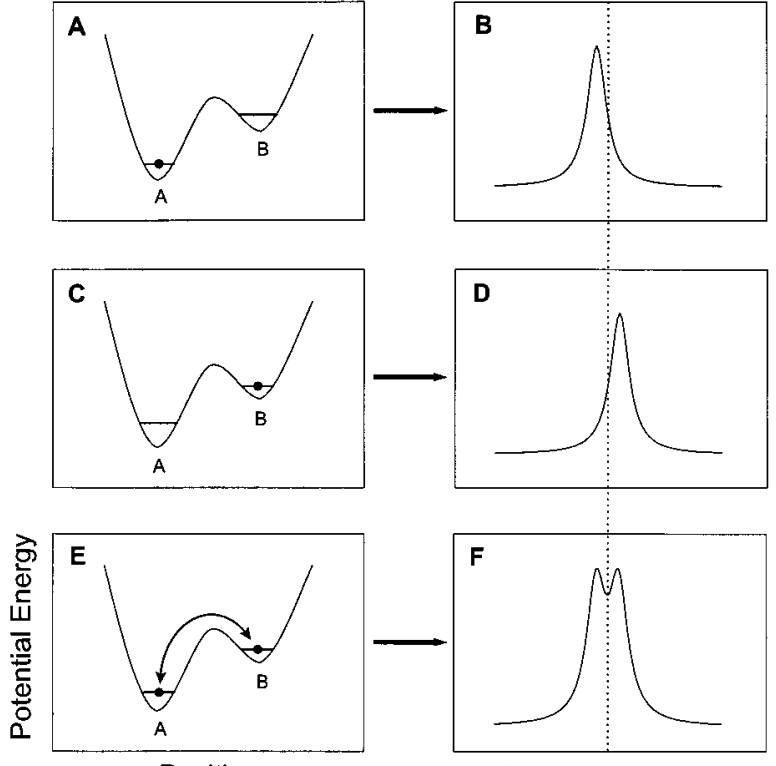

Position
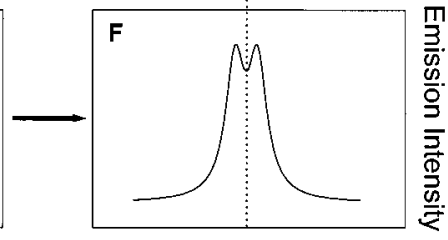

Emission Energy
Figure 5. (A) and (B) Potential energy curve and resulting single dot spectrum for a charge trapped in the left half of a double welled potential. (C) and (D) Same as (A) and (B) with the charge trapped in the right-hand well. (E) Potential energy diagram in which the charge can freely move between the wells so that the observed single dot spectrum is dependent on the relative occupancy of each well. (F) The resulting single dot spectrum if the charge in (E) were to spend approximately equal time in each trap (this is the "high-energy" limit where the available thermal and excess excitation energy is much larger than the difference in energy between the two wells). The emission energy of the peaks in Figure 5B and D were arbitrarily chosen and are not expected to relate, in a direct way, to the energy of the corresponding trap site.

tions/s with $\Delta T$ for the nanocrystallite $\approx 6 \mathrm{~K} / \mathrm{excitation}$, which dissipates in $<10^{-12} \mathrm{~s}^{35}$ ). Instead, phonons emitted as the exciton relaxes to its lowest excited state may couple directly to trapped charges. Following each excitation, there is a certain probability that an external charge will overcome the potential barrier between adjacent trap sites as a result of the released energy. The more often the dot is excited, the more chances a charge has to escape and the more changes in the local field configuration can occur. The result is a broadening of the observed single dot spectrum as time or intensity is increased. Saturation occurs when spectral diffusion has reached a steady state condition. At this point, the line width is no longer dependent on the kinetics of charge movement, but only on the relative probability that the QD has experienced each available charge configuration. The saturation line width is then a function of the equilibrium distribution of trap occupancies and will therefore depend on the number and type of external charges, the relative energies of each trap site, and the available thermal and excitation energy. To illustrate the effect of changing temperature or excitation energy on a single dot spectrum, consider the simple case of a two-trap system, containing a single charge (Figure 5). In this case, the equilibrium occupancy of sites $\mathrm{A}$ and $\mathrm{B}$ ([A] and [B]) can be expressed as an exponential function of the available thermal and excess excitation energy. If we assume that the local electric field experienced by the QD depends on the location of the trapped charge, with the resulting spectra shown in Figure 5B and D, then the saturation line width of a single dot spectrum depends on the relative amount of time that the charge spends in each trap. When the available thermal and excitation energy is small compared to the difference in energy between trap A and B,

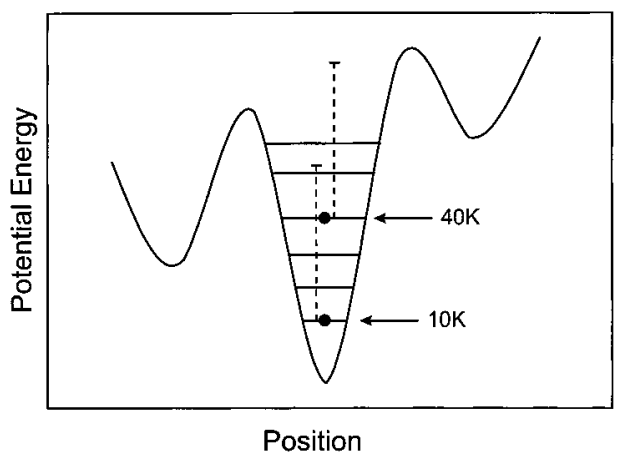

Figure 6. Thermally assisted charge movement. A graphic representation of a charge in a trap site at two different temperatures. Dotted lines indicate the energy added to the system from excess excitation energy.

then $[\mathrm{B}] \ll[\mathrm{A}]$ and the saturation line width is dominated by the contribution from site $\mathrm{A}$. In this case, the observed spectrum is that of Figure $5 \mathrm{~B}$. When the available energy is high relative to the energies of $\mathrm{A}$ and $\mathrm{B}$, then $[\mathrm{B}] \approx[\mathrm{A}]$ and the observed saturation line width is that of Figure $5 \mathrm{~F}$. Increasing the amount of available energy not only increases the saturation line width but also increases the rate at which saturation is reached. From this simple example, it is clear that both the slope and saturation line width of a single dot spectrum should be strongly dependent on the amount of available energy. Figure 2 demonstrates that this is the case for increasing temperature.

At very short times the number of changes in the local electric field becomes small and spectra should approach an intrinsic width. Consistent with this, individual single dot line widths become very narrow as the integration time approaches zero (Figure 4). Similar results are also observed at low excitation intensity, consistent with the proposed model.

The thermal broadening observed in Figure 2 is not the result of coupling of the emitting state to acoustic phonons. Figure 1 demonstrates that under normal conditions, broadening due to dephasing is insignificant relative to the contribution of spectral diffusion. Similar to time and intensity, the effect of temperature can be explained in terms of spectral diffusion, as described above (Figure 5). The overlap of the time and intensity curves in Figure 2, plotted as a function of excitation energy density, however, indicates that broadening as a direct result of thermally activated movement of external charge carriers is insignificant in this temperature range. Broadening due to direct thermal motion would result in a divergence of the time and intensity curves, with the time curve saturating more quickly than the intensity curve. This is not observed. At room temperature, however, the average line width of 31 overcoated dots $(67 \mathrm{meV}$, $\sigma=18 \mathrm{meV}$ ) was found to be independent of excitation intensity, suggesting that direct thermal movement is the dominant broadening effect at room temperature.

The data in Figure 2 are consistent with a thermally assisted process. What is meant by "assisted" is that while thermal energy alone may not result in significant spectral broadening, the exponential dependence of the escape probability from a given trap site implies that the total available energy (thermal + excess excitation) can have a much larger effect than either one alone. As the temperature is increased, trapped external charges populate a higher energy within each potential well (Figure 6). Following each excitation, a higher energy charge has a greater probability of escaping a given trap. The observed thermal broadening is then the result of a larger number of excitations that result in a successful change in the local field configuration. 
It is important to point out that it is the change in the local electric field resulting from the movement of charges between trap sites, and not the relative energies of the traps, that determines the magnitude of a spectral diffusion shift. As a result, there is not necessarily a correlation between the energy difference between trap sites and the magnitude of the resulting spectral diffusion shift when a charge moves between these sites. While it may be possible to model the movement of charges between trap sites as an exponential function of the available energy, the resulting line width (the observable measured in these experiments) does not necessarily follow such a simple behavior since it also depends on the relative location of each trap site. Also, it should be noted that while the number of potential trap sites around a QD must be finite, the fact that multiple charges can contribute to the local field may result in a quasicontinuous distribution of potential field configurations.

While the experiments described in this Article were performed on nanocrystallite QDs, the spectral diffusion effects observed may also be relevant in other QD systems. Line widths in single GaAs QDs formed by potential fluctuations in thin quantum wells are found to be significantly broader for dots close to the surface of the QW structure. ${ }^{15}$ This broadening in the presence of an exposed surface where charges can reside is consistent with spectral diffusion and the effects described above.

In conclusion, we have demonstrated the role of spectral diffusion in the observed line shapes of single CdSe nanocrystallite QDs. Data suggest that single QD line shapes are primarily the result of light-driven spectral diffusion. These spectral shifts occur on a time scale that is fast relative to the acquisition time of the experiment and are activated by excess energy released following each photoexcitation. While thermal effects are found to play an indirect role in line widths at temperatures below 40 $\mathrm{K}$, they become a dominant effect at room temperature. Under normal conditions, the shape of a single nanocrystallite spectrum contains information about the dynamics of the local environment and not the inherent physics of the nanocrystallite. These data suggest that it is inappropriate to attempt to define a "homogeneous" line shape or width for these nanocrystallites on the time scale of fluorescence measurements. Instead, single dot line widths serve only as an upper bound for the true intrinsic width.

Acknowledgment. This work was funded in part by the NSF-MRSEC program (DMR-94-00334) and the AT\&T foundation. We thank the MIT Harrison Spectroscopy laboratory (NSF-CHE-93-04251) for support and for use of its facilities.

\section{References and Notes}

(1) Lu, H. P.; Xun, L.; Xie, X. S. Science 1998, 282 (5395), 1877.

(2) Lu, H. P.; Xie, X. S. J. Phys. Chem. B 1997, 101, 2753.

(3) Ha, T.; Glass, J.; Enderle, Th.; Chemla, D. S.; Weiss, S. Phys. Rev. Lett. 1998, $80(10), 2093$.

(4) Nirmal, M.; Dabbousi, B. O.; Bawendi, M. G.; Macklin, J. J.; Trautman, J. K.; Harris, T. D.; Brus, L. E. Nature 1996, 383, 802.

(5) Basche, T. J. Lumin. 1998, 76 \& 77, 263.
(6) Dickson, R. M.; Cubitt, A. D.; Tsien, R. Y.; Moerner, W. E. Nature 1997. $399,255$.

(7) Ambrose, W. P.; Basche, T.; Moerner, W. E. J. Chem. Phys. 1991, 95 (10), 7150.

(8) Tchenio, P.; Myers, A. B.; Moerner, W. E. J. Lumin. 1993, 56, 1. (9) Empedocles, S. A.; Norris, D. J.; Bawendi, M. G. Phys. Rev. Lett. 1996, 77 (18), 3873.

(10) Blanton, S. A.; Dehestani, A.; Lin, P. C.; Guyot-Sionnest, P. Chem. Phys. Lett. 1994, 229, 317. Blanton, S. A.; Hines, M. A.; Guyot-Sionnest, P. Appl. Phys. Lett. 1996, 69 (25), 3905.

(11) Tittel, J.; Gohde, W.; Koberling, F.; Basche, Th.; Kornowski, A.;

Weller, H.; Eychmuller, A. J. Phys. Chem. B 1997, 101 (16), 3013.

(12) Empedocles, S.; Bawendi, M. Science 1997, 278, 2114.

(13) Norris, D. J.; Sacra, A.; Murray, C. B.; Bawendi, M. G. Phys. Rev. Lett. 1994, 72 (16), 2612

(14) Murray, C. B.; Norris, D. J.; Bawendi, M. G. J. Am. Chem. Soc. 1993, 115 (19), 8706

(15) Hess, H.; Betzig, E.; Harris, T. D.; Pfeiffer, L. N.; West, K. W. Science 1994, 264, 1740. Gammon, D.; Snow, E.; Katzer, D. Appl. Phys. Lett. 1995, 67, 2391.

(16) Brown, S. W.; Kennedy, T. A.; Gammon, D.; Snow, E. S. Phys. Rev. B 1996, 54 (24), R17339.

(17) Landin, L.; Miller, M. S.; Pistol, M. E.; Pryor, C. E.; Samuelson, L. Science 1998, 280, 262

(18) Dekel, E.; Gershoni, D.; Ehrenfruend, E.; Spektor, D.; Garcia, J. M.; Petroff, P. M. Phys. Rev. Lett. 1998, 80 (22), 4991.

(19) Gammon, D.; Snow, E. S.; Shanabrook, B. V.; Katzer, D. S.; Park, D. Science 1996, 273 (5271), 87.

(20) Woggon, U.; Gaponenko, S.; Langbein, W.; Uhrig, A.; Klingshirn, C. Phys. Rev. B 1993, 47, 3684

(21) Mittleman, D. M.; Schoenlein, R. W.; Shiang, J. J.; Colvin, V. L.; Alivisatos, A. P.; Shank, C. V. Phys. Rev. B 1994, 49 (20), 14435.

(22) Norris, D. J.; Bawendi, M. G. J. Chem. Phys. 1995, 103 (13), 5260; Phys. Rev. B 1995, 53, 16338 .

(23) Alivisatos, A. P.; Harris, A. L.; Levinos, N. J.; Steigerwald, M. L.; Brus, L. E. J. Chem. Phys. 1988, 89 (7), 4001.

(24) Jungnickel, V.; Henneberger, F. J. Lumin. 1996, 70, 238

(25) Nirmal, M.; Norris, D. J.; Kuno, M.; Bawendi, M. G. Phys. Rev. Lett. 1996, 75, 3728

(26) Norris, D. J.; Bawendi, M. G. Phys. Rev. B 1996, 53 (24), 16338

(27) Hessman, D.; Castrillo, P.; Pistol, M. E.; Pryor, C.; Samuelson, L. Appl. Phys. Lett. 1996, 69 (6), 749. Steffen, R.; Forchel, A.; Reinecke, T. L.; Koch, T.; Albrecht, M.; Oshinowo, J.; Faller, F. Phys. Rev. B 1996, 54 (3), 1510.

(28) Trautman, J. K.; Macklin, J. J.; Brus, L. E.; Betzig, E. Nature 1994, 369,40 .

(29) Geva, E.; Skinner, J. L. J. Phys. Chem. B 1997, 101 (44), 8920 Pfluegl, W.; Brown, F. L. H.; Silbey, R. J. J. Chem. Phys., in press.

(30) Hines, M. A.; Guyot Sionnest, P. J. Phys. Chem. 1996, 100, 468 Dabbousi, B. O.; Rodriguez-Viejo, J.; Mikulec, F. V.; Heine, J. R. Mattoussi, H.; Ober, R.; Jensen, J. F.; Bawendi, M. G. J. Phys. Chem. B 1997, 101, 9463.

(31) Peaks in Figure 1B (frames 1-5) represent $\sim 20$ collected photons, indicating that changes in emission energy are not statistical fluctuations in single photon energies.

(32) Efros, Al. L.; Rosen, M. Phys. Rev. Lett. 1997, 78 (6), 1110.

(33) Norris, D. J.; Sacra, A.; Murray, C. B.; Bawendi, M. G. Phys. Rev. Lett. 1994, 72 (16), 2612.

(34) Emission intensity was used as a measure of excitation rate. The excitation intensity was adjusted so that the average emission intensity was the same at both wavelengths. Since quantum yield has been found to be relatively wavelength independent in the region that we are exciting, each photon emitted should correspond to a fixed number of photons absorbed. This procedure should allow us to normalize the excitation rate. The average emission intensity for $573 \mathrm{~nm}$ excitation was actually slightly higher $(\sim 8 \%)$ than that for $514 \mathrm{~nm}$, indicating that the observed decrease in line width was not related to the number of excitations.

(35) Dissipation time is estimated from the rate of heat transfer to a thermally conducting sphere with a constant surface temperature. [Carslow, H. S.; Jaeger, J. C. Conduction of Heat in Solids; Oxford University Press: Oxford, U.K., 1947)] 